Review

\title{
Towards Deep Integration of Electronics and Photonics
}

\author{
Ivan A. Pshenichnyuk ${ }^{1, * \mathbb{D}}$, Sergey S. Kosolobov ${ }^{1}(\mathbb{D})$ and Vladimir P. Drachev ${ }^{1,2, * \mathbb{D}}$ \\ 1 Skolkovo Institute of Science and Technology, Center for Photonics and Quantum Materials, Nobelya 3, \\ 121205 Moscow, Russia; S.Kosolobov@skoltech.ru \\ 2 Department of Physics, University of North Texas,1155 Union Circle, Denton, TX 76203, USA \\ * Correspondence: I.Pshenichnyuk@skoltech.ru (I.A.P.); Vladimir.Drachev@unt.edu (V.P.D.)
}

Received: 15 September 2019; Accepted: 5 November 2019; Published: 12 November 2019

\begin{abstract}
A combination of computational power provided by modern MOSFET-based devices with light assisted wideband communication at the nanoscale can bring electronic technologies to the next level. Obvious obstacles include a size mismatch between electronic and photonic components as well as a weak light-matter interaction typical for existing devices. Polariton modes can be used to overcome these difficulties at the fundamental level. Here, we review applications of such modes, related to the design and fabrication of electro-optical circuits. The emphasis is made on surface plasmon-polaritons which have already demonstrated their value in many fields of technology. Other possible quasiparticles as well as their hybridization with plasmons are discussed. A quasiparticle-based paradigm in electronics, developed at the microscopic level, can be used in future molecular electronics and quantum computing.
\end{abstract}

Keywords: plasmon; light-matter interaction; photonics; nano-electronics; quasiparticle

\section{Introduction}

Further achievements in electronics and photonics imply their better integration. Usage of photons at the level of circuits and single chips has a huge potential to improve modern intelligent systems [1]. Along with the usage of light for communication at small and intermediate scales, photonic circuits may also perform logical and computational operations. This implies that the distribution of functions between photons and electrons may be more advanced and potentially more productive than just communication and computation in the future generation of electro-optical chips. Regarding the future of electronics, it is important to note that the development of modern nanofabrication techniques and mesoscopic physics unavoidably leads to molecular electronics, where basic elements of circuits are represented by single molecules [2]. The idea to use both electrons and photons in future "molecular chips", operated by a microscopically small amount of particles, requires the detailed understanding of their quantum properties and interactions.

The diffraction limit, unbeatable in classical photonics, keeps the size of light processing components at least an order of magnitude larger than the size of the electrical counterpart, i.e., transistors. The typical size of a transistor in modern processors is of the order of $10 \mathrm{~nm}$, while the telecom wavelength of light is close to $1.5 \mu \mathrm{m}$. In classical photonics, the electro-optical effect enables a modulation of optical signals due to changes in the optical parameters of materials under applied electric field. This effect is typically not as strong to use it on a chip. The mismatch in dimensions and weak light-matter interaction motivates a search for novel materials and subwavelength optics usage [3]. 
A plasmon is defined as a quantum of collective electron oscillation in a solid. It is also referred as a bulk plasmon (Figure 1a). It may reveal itself in metals, doped semiconductors and other materials (see the next section), where the concentration of charge carriers is sufficient. Charge oscillations near metallic surfaces can be coupled with light, forming surface plasmon polaritons (SPP) [4-6] as illustrated in Figure 1b. The localized surface plasmons (LSP) are typical for metallic nanoparticles and rods, where they may also be called transverse plasmons (see Figure 1c). The plasmon observations can be done with electron energy loss spectroscopy (EELS) with excitation by electrons [7], in the extinction optical spectra of small particles and films [8,9], or cathodeluminescence (see [5] and references there). At present time the phenomena of light-matter coupling at the interface between metals and dielectrics is well understood [10]. Traditional applications of LSPs include surface-enhanced Raman spectroscopy (SERS) [11,12], sensing [13,14], scanning near-field optical microscopy (SNOM) and tip-enhanced Raman scattering (TERS) (see [4] and references therein). The SPP modes attract an interest from the point of view of communication due to their subwavelength confinement. This property of SPPs can be extremely useful for applications and allows to bring electronics and photonics closer to each other [3,15-19].

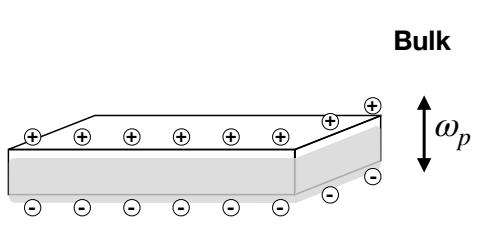

(a)

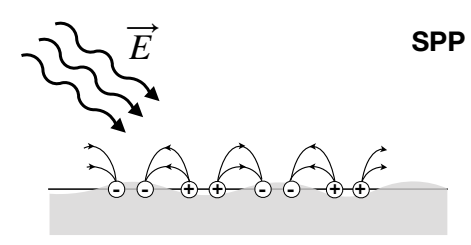

(b)

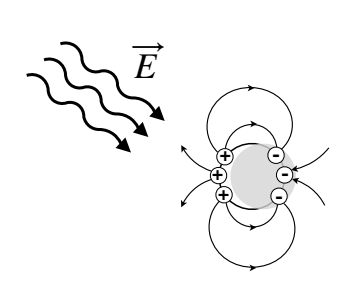

LSP

(c)

Figure 1. Various types of plasmonic excitations: (a) Bulk plasmon in a metallic sample, electronic gas oscillates as a whole with a plasmonic frequency $\omega_{p}$. (b) Propagating surface plasmon polariton excited by the external electromagnetic wave. (c) Localized surface plasmon excited by the external field in a small metallic nanoparticle. Displacement of the electron gas is shown with light grey.

A key active component in the interaction between electronic and photonic subsystems in mixed circuits is a modulator, i.e., the device which converts an electronic signal to an optical signal or vice versa. The importance of this active element is comparable with the importance of transistors in electronics. While pure electronic or pure optical transistors may perform logical, computational and information processing operations, modulators allow two subsystems talking to each other. Plasmonic modes discussed above can be used to make such devices small and efficient [20]. Various types of electro-optical modulators are discussed below along with other important components required to build fully integrated circuits, like waveguides, light sources, polarization converters and amplifiers. It is remarkable that most of the required components can be improved using plasmonics. The discussion of building blocks of hybrid circuits in Section 3 of this paper is preceded by the discussion of perspective plasmonic materials in Section 2. Key experiments and achievements in the field of quantum plasmonics are presented in the Section 4. A brief discussion of other quasiparticles capable of light-matter hybridization as well as their interaction with plasmons is presented in Section 5 . The conclusion follows in Section 6. 


\section{Perspective Materials}

Traditional plasmonic materials are gold and silver due to the fact that their relaxation constants are less than for other metals (approximately $21 \mathrm{meV}$ for silver and $66 \mathrm{meV}$ for gold [21]). The SPP modes are supported at the interface between dielectrics and noble metals due to the large negative real part of the permittivity of the latter [4]. Metals introduce optical losses, caused by the imaginary part of the permittivity. The resulting damping of surface plasmons limits their practical application. Extra problems are related to interband transitions which result in additional losses. These factors stimulate the search for alternative plasmonic materials with improved properties and the extension to the near-infrared (NIR) including the telecom spectral range [22,23]. Spectral coverage of plasmonic materials were extended also into the deep ultraviolet (UV), where unique plasmon properties have been discovered for ferromagnetic materials as a result of spin-polarization [24]. Alternative plasmonic materials with desired properties applicable in nanophotonics include semiconductors, transparent conductive oxides (TCO) and 2D materials.

The excitation of a SPP requires to reach a certain density of charge carriers. Appropriately doped semiconductors, like Si and GaAs may support plasmonic modes. Silicon plasmonics is discussed in the literature as a platform for on-chip photonics with the SPP propagation length of the order of ten micrometers $[25,26]$. The advantage of $\mathrm{Si}$ is that it is the most common material in electronics with well-developed manufacturing and processing technologies. However, it requires a relatively high level of doping $\left(10^{21} \mathrm{~cm}^{-3}\right)$ to reveal plasmonic properties at telecommunication wavelengths [23].

Another class of materials, commonly referred as transparent conductive oxides, is proven to be useful in plasmonics. Traditional representatives of this class are aluminum-doped zinc oxide (AZO), gallium-doped zinc oxide (GZO) and tin-doped indium oxide (ITO). TCOs show good plasmonic properties in the IR [27]. These materials allow high doping, which makes them conductive [28,29]. At the same time, a large band gap makes them transparent in the visible range. Materials such as ITO are suitable to produce thin films and are compatible with silicon-based components. The excitation of SPPs in ITO has been demonstrated experimentally in the NIR range [30,31]. A relatively low external voltage (below $10 \mathrm{~V}$ ) applied to ITO can induce a refractive index change of about one $(\Delta n \sim 1)$ at telecom wavelengths, which is a desired property in switches and modulators [32].

According to the Drude model, at optical frequencies $\omega$ permittivity reads

$$
\varepsilon(\omega)=\varepsilon_{\infty}-\frac{\omega_{p}^{2}}{\left(\omega^{2}+\gamma^{2}\right)}+i \frac{\gamma \omega_{p}^{2}}{\omega\left(\omega^{2}+\gamma^{2}\right)}
$$

where $\omega_{p}=\sqrt{n_{c} e^{2} /\left(\varepsilon_{0} m\right)}$ is a plasma frequency, $\gamma$-relaxation coefficient, $\varepsilon_{\infty}$-asymptotic permittivity at large frequencies, $e$-electron charge, $m$-effective electron mass, $\varepsilon_{0}$-free space permittivity and $n_{c}$ denotes the concentration of charge carriers. The real part of the permittivity may cross zero at certain wavelengths and charge densities. The imaginary part, at the same time, remains finite, which excludes singularities in Maxwell equations. Nevertheless, this epsilon-near-zero (ENZ) point is quite special, since properties of materials vary significantly there [33]. As it was shown experimentally, the Drude model describes ITO quite accurately [34] and the ENZ point is in the IR spectral range. Since the permittivity of ITO depends significantly on the free charge density which enters the plasma frequency, one can locally shift the ENZ wavelength using an external electric field or applying a voltage. The realization of this mechanism in electro-optical devices requires the incorporation of ITO/dielectric capacitors, suitable for charge accumulation. Another approach is to mix TCOs with noble metals to obtain hybrid plasmonic structures with desired properties. For instance, Ag-ITO co-sputtered composite films [35] and thin Au spacer layers incorporated inside ITO films [36] allow to control the ENZ-point wavelength and thus provide better tunability of the system. The development and structuring of metallic/dielectric composites leads to the idea of plasmonic metamaterials supporting the ENZ effect with many intriguing properties, like, for example, optical nonlocality [37]. 
Surface plasmons can be excited in 2D materials as well. One of the most studied examples is graphene. Optical properties of such structures are obviously qualitatively different from 3D bulk materials or films. The dispersion relation of massless Dirac electrons and its influence on the overall material properties are well known. Plasmonic behavior of graphene can be engineered using geometrical variations, doping and stacking [38,39]. Thus, one of the characteristic features of 2D structures is high tunability. Gating works particularly well there [40]. It is experimentally demonstrated that in graphene $/ \mathrm{SiO}_{2} / \mathrm{Si}$ structures it is possible to alter both the amplitude and wavelength of plasmons by applying a gate voltage [41]. Typical propagation length of the order of micrometers (several plasmon wavelengths) is reported in these experiments. Fast and efficient gating leads to various applications including graphene-based optical transistors and modulators [42]. Fundamental limits of graphene plasmonics due to losses were probed recently in cryogenic experiments, revealing the physics of plasmonic dissipation [43]. At the temperature of liquid nitrogen, when certain mechanisms of dissipation can be excluded, the plasmon propagation length grows and can exceed $10 \mu \mathrm{m}$ (approximately 50 wavelengths).

High quality dielectrics are among auxiliary materials which are important in plasmonic applications [44,45]. One good example is hafnium dioxide, which was introduced by Intel as an advanced MOSFET gate insulator about a decade ago [46]. Materials like $\mathrm{HfO}_{2}$ with the static permittivity approximately 6 times larger than silica allow to significantly decrease the operating voltage and energy consumption of the resulting devices.

\section{Building Blocks of Hybrid Integrated Circuits}

The building blocks of modern electronic circuits are well established. Components of emerging nanophotonic circuits are usually sorted as the devices that generate light, guide, manipulate, and detect light. Both electronic and photonic subsystems in mixed circuits may contain active elements, like transistors, which are key elements for computations and information processing. A modulator allows manipulation of a photonic signal using an electronic signal or vice versa. Plasmonics is considered here as a promising tool to make the light-matter interaction stronger and corresponding devices compact and fast.

Regarding the plasmonic improvements in the area of light emission, it is worth mentioning the technique that enhances the light-emitting efficiency of LEDs [47]. In these experiments, a supplementary silver layer is deposited $10 \mathrm{~nm}$ above the InGaN quantum well LED structure. According to the results, coupling between SPP, supported by the metallic layer, and the well increases the density of states in a semiconductor and enhances the intensity of light emission [47]. This effect can be used to construct more efficient solid-state light sources. The interaction between SPPs and semiconductors raises the fundamental question of coupling between excitons and SPPs. There are many applications of this mechanism, including a single photon SPP quantum detector and plasmonic photovoltaics (see below).

Plasmonic waveguiding allows to reach a large degree of confinement in comparison with ordinary dielectric diffraction limited waveguides (Figure 2). In the case of a silicon at the typical telecom frequencies, a minimal waveguide cross section supporting a single mode is about $400 \mathrm{~nm} \times 200 \mathrm{~nm}$. Plasmonic modes can be confined in layers with thicknesses of the order of tens of nanometers [48]. Thus, subwavelength plasmonic waveguides combine compactness of electronic circuits with a wide band communication capability, typical for photonic networks. The simplest plasmonic waveguide, supporting SPP modes, is just a metal-dielectric interface (Figure 2a). Another convenient structure, especially in quantum plasmonics, is a metallic nanowire [49] (efficient experimental techniques exist to produce thin nanowires with a large degree of homogeneity, see below). Various types of SPP-supporting geometries were suggested in the literature, like metal-insulator-metal and insulator-metal-insulator sandwiches, dielectric loaded plasmonic waveguides, gap waveguides and many others [19]. A convenient feature of the plasmonic waveguiding concept is that metallic surfaces, which already exist in electronic chips, may be used for SPP guiding. For example, metallic contacts in 
circuits or gate electrodes in transistors can be used as plasmonic waveguides, allowing to naturally incorporate SPP communication into circuits. Losses introduced by metals limit the utility of plasmonic waveguides and complicate the realization of a plasmonic communication network. Nevertheless, for some applications, like, for example, short distance communication between transistors, SPP propagation length provided by ordinary gold/silver surfaces is sufficient.

To control plasmonic losses, an elegant solution exists in the form of so-called hybrid plasmonic waveguides (HPWG) [50]. The idea is to combine an ordinary lossless nanoscale photonic waveguide with poor mode confinement and a lossy but compact plasmonic waveguide (Figure 2c). As a result of interaction, a hybrid mode appears which represents a compromise between confinement and losses. By varying the distance between waveguides and the corresponding interaction strength, one may choose a desired proportion. Usually the interaction is strong, which makes the hybrid mode behaviour complicated and requires numerical computations to analyze its propagation. The concept of HPWG is valuable for plasmonic electro-optical modulators, since it allows to make them much smaller (see below). Many other components implementing this principle were suggested, like polarization converters, directional couplers, splitters and ring resonators [50].

\section{Plasmonic waveguide}

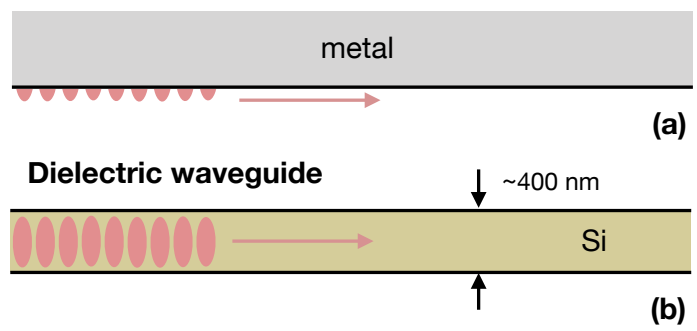

Hybrid plasmonic waveguide

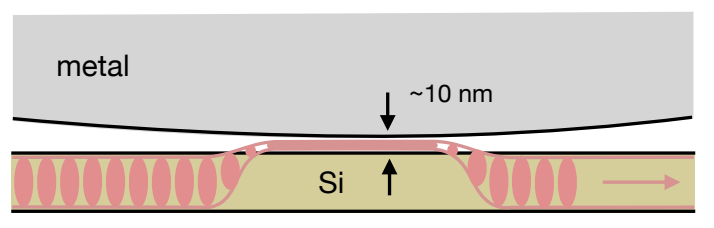

(c)

Figure 2. Combining a plasmonic waveguide (a), represented by a metallic surface, with a dielectric waveguide (b) one can form a hybrid plasmonic waveguide (c). A hybrid mode evolution is accompanied by a periodic energy transfer between the dielectric waveguide and the gap. Choosing the right length and distance one can use this effect to perform a smooth conversion of the waveguide mode into plasmon and back. A similar mechanism in successfully used in various models of plasmonic electro-optical modulators.

Another way to overcome plasmonic losses is to use gain medium and amplifiers. A theoretical explanation of the gain medium mechanism to compensate SPP losses is given in [51]. Performing finite element simulations, the authors demonstrate the possibility of lossless propagation of SPP on a silver surface adjacent to InGaAsP-based medium. SPP amplification is realized experimentally [52] using the Kretschmann scheme, where the silver surface is sustained in contact with optically pumped dye-based gain medium. The achieved value of gain is sufficient to fully compensate losses in silver films. Gain-assisted propagation in plasmonic waveguides is experimentally studied in [53]. A single mode plasmonic waveguide is fabricated using a polymer (polymethylmethacrylate) strip placed on a gold surface. To compensate losses, the waveguide is doped by lead sulfide ( $\mathrm{PbS}$ ) quantum dots. Laser pumping in the central part of the waveguide results in a stimulated emission and increases the propagation length of SPP mode by 27 percent at telecom wavelengths. The direct measurement of SPP gain in a symmetric gold-stripe waveguide was performed in the experiments with optically pumped gain medium, represented by dye molecules in a solution [54]. Mode power gain of $8.55 \mathrm{~dB} / \mathrm{mm}$ is achieved. By combining gain medium with a plasmonic resonator (both SPP and LSP modes can be used) one obtains SPASER (surface plasmon amplification by stimulated emission of radiation) [55]. Nanoparticles wrapped with a gain medium, for instance, can be used to construct about $20 \mathrm{~nm}$ large super-bright light sources useful in biomedical applications [56]. 
While light sources and waveguides allow to form a photonic network, electro-optical modulators are responsible for the interaction with electronic components of the circuits. From the applied point of view, the major question is how to organize the interaction in the most efficient way. Many theoretical [57-62] and experimental [63-68] research works were focused on the models of plasmonic modulators. The HPWG concept and ENZ effect described above are two of the most promising plasmonic solutions which are implemented in different forms and combinations in these models. Different realizations compete for better energy consumption, bandwidth, size and CMOS-compatibility.

The idea of a plasmostor is proposed and realized experimentally in [64] (see Figure 3a). In the suggested design, the distance between two silver electrodes is filled by $170 \mathrm{~nm}$ thick doped silicon and $10 \mathrm{~nm}$ thick insulating layer of $\mathrm{SiO}_{2}$. Voltage applied to the electrodes influences properties of two available modes, allowing them to interact constructively or destructively at the output. Doped silicon does not support ENZ regime at telecom frequencies, but still may act as an active material, changing its refractive index under the applied voltage. Interference between two modes, thus, provides the modulation effect. There are other prototypes of modulators where the plasmonic slot waveguide configuration is implemented [57] (see Figure 3b). A thin layer of ITO $(10 \mathrm{~nm})$ is used in this model as an active material combined with a $50 \mathrm{~nm}$ thick layer of $\mathrm{SiO}_{2}$. Under the applied voltage, additional charges are accumulated in ITO, changing its local permittivity, both real and imaginary parts, and providing the absorption-based modulation. The refractive index of ITO changes much stronger at the applied voltage, compared with Si. In this model the ENZ effect is not used. Further development of the plasmonic slot modulator idea appears in the experiments of [66] (see Figure 3c). The $300 \mathrm{~nm}$ space between gold electrodes is filled by ITO, separated from the surface of the metal by a thin layer of $\mathrm{Al}_{2} \mathrm{O}_{3}$ insulator. The resulting capacitor allows to accumulate reasonable charges at the $\mathrm{ITO} / \mathrm{Al}_{2} \mathrm{O}_{3}$ interface at relatively small voltages and reach the ENZ effect in ITO, providing an efficient modulation.

In classical photonic circuits, light is assumed to be confined in a dielectric low-loss waveguide. In this case, the application of modulators described above requires the waveguide mode to be transfered into a plasmonic slot mode and back, with unavoidable transformation losses. A fully waveguide-based plasmonic modulator is demonstrated in [68] (see Figure 3d). In this work, the modulating sandwich consisting of layers of ITO $(10 \mathrm{~nm}), \mathrm{SiO}_{2}(20 \mathrm{~nm})$ and $\mathrm{Au}$ is placed on top of a silicon waveguide. A gold layer is used as both an electrical contact and a plasmonic surface. Conversion of a waveguide mode into a hybrid modulator mode (like in Figure 2c) can be made smooth and effective in such a model, by appropriately adjusting the geometry. The applied voltage shifts the frequency of the ENZ point in ITO, effectively absorbing the localized hybrid mode. This structure thus uses both the HPWG and the ENZ effect described above, resulting in a fast and compact model of electro-optical modulator.

A family of modulators exists where only the ENZ effect is used to provide switchable absorption with no propagating plasmonic modes involved. Usually, such an approach makes the resulting devices longer in size compared to HPWG based solutions. In the theoretical work [69] (see Figure 3e) an active layer of AZO is incorporated into a single-mode silicon waveguide. In the off-state (when the voltage is applied) an absorbing layer is formed in the middle of the waveguide core, where the intensity of the propagating mode is maximal, providing an efficient modulation. In calculations of [60] (see Figure 3f) and [59] it is suggested to place ITO either around the waveguide or allow it to partially penetrate inside, realizing a similar modulation principle. On the other hand, fully metallic modulators were suggested recently which use only propagating plasmonic modes along with the Mach-Zehnder interference mechanism [63]. To compare with ENZ modulators, metallic solutions usually introduce more optical losses. Instead of fighting these losses, some authors suggest how to use them, for example, allowing SPP modes to come into play only in the off-state of the modulator, where losses work in a positive way [65] More details and an extensive discussion on the performance and characteristics of plasmonic modulators are available in multiple reviews $[20,26,70]$. 

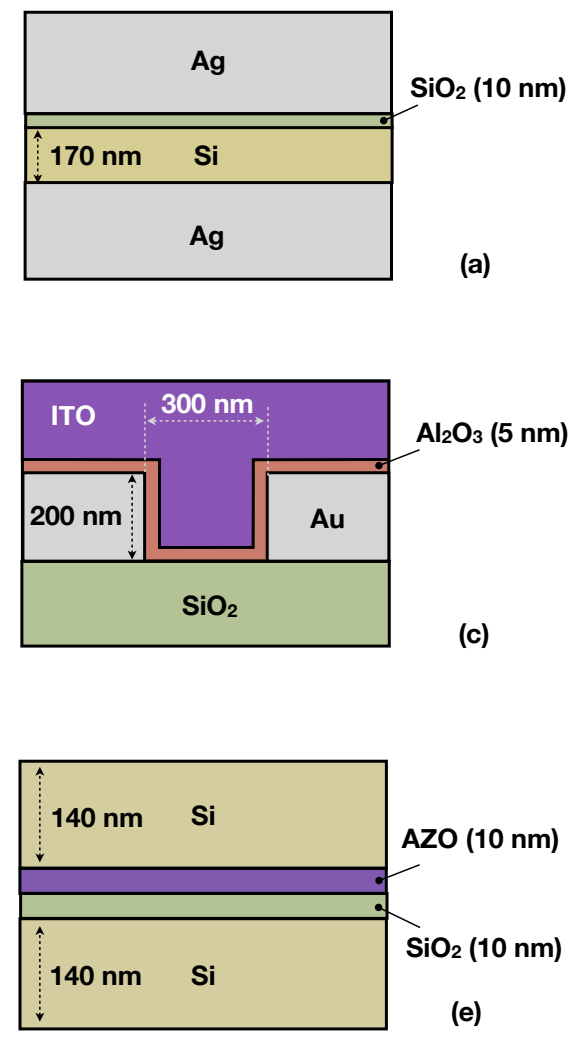
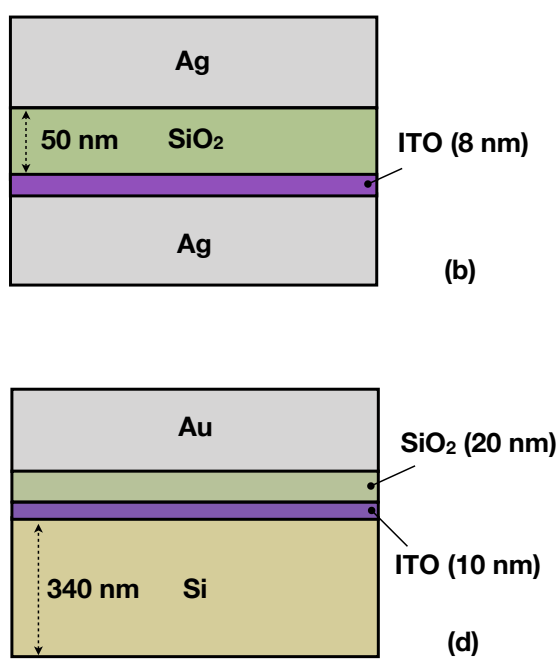

(d)

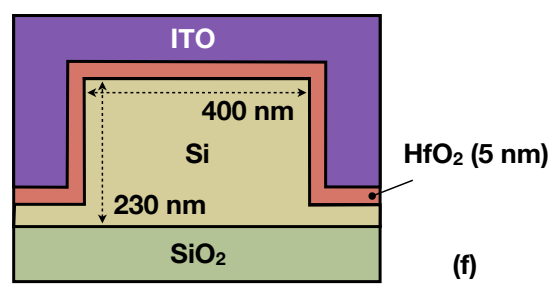

Figure 3. Various models of electro-optical modulators available in literature: (a) Silicon field effect plasmostor. Reprinted with permission from [64]; 2009 American Chemical Society. (b) Surface plasmon polariton absorption modulator. Reproduced from [57] with the permission. (c) Nanoscale conducting oxide plasmostor. Reprinted with permission from [66]; 2014 American Chemical Society. (d) Ultra-compact silicon nanophotonic modulator. Reproduced from [68] with the permission. (e) Ultra-compact electroabsorption modulator based on tunable ENZ waveguides. Reproduced from Lu et al. [69] with the permission. (f) TCO electro-optical modulator on silicon platform. Reproduced from [60], with the permission of AIP Publishing. In all cases the direction of optical modes propagation is orthogonal to the plane of the figure.

SPP-based plasmonic modulators are usually strongly polarization-dependent. This is the consequence of the fact that surface plasmons polaritons can be excited only by light polarized perpendicularly to the surface of a metal. For this reason, another important class of auxiliary devices in photonic circuits are polarization converters. Many solutions with different levels of compactness and structural complexity were suggested [71-73]. Some of these devices may have a structure very similar to the structure of modulators [74] suggesting the idea to combine both devices into one universal unit. In recent experiments, a plasmonic mechanism of conversion is demonstrated [75]. Gold impurity is asymmetrically integrated into the waveguide. The evolution of a hybrid mode is accompanied by the transformation of polarization. The question of conversion efficiency in such devices becomes crucial for large circuits, where optical losses introduced by each device are summed up.

The construction of large plasmonic circuits using the developed structural elements is still at the initial phase [19]. Chip-to-chip data transfer using SPP is demonstrated experimentally [76]. Four-channel array of plasmonic waveguides made of $2.5 \mathrm{~cm}$ long golden strips with a polymer cladding is used. A $10 \mathrm{Gbps}$ transmission rate is reached (2.5 Gbps for each channel) at the wavelength of $1.3 \mu \mathrm{m}$. A plasmonc circuit for optical signal processing is experimentally demonstrated by [77]. The proposed structure represents a $200 \mathrm{~nm}$ large ensemble of metallic nanorods supporting evanescently coupled LSP. The device mixes two optical signals and provides a measure of their phase difference at the output. It is claimed that similar circuits can perform other mathematical operations on multiple signals. 
The construction of large plasmonic circuits using the developed structural elements is still in the initial phase [19]. Chip-to-chip data transfer using SPPs has been demonstrated experimentally [76]. A 4-channel array of plasmonic waveguides made of $2.5 \mathrm{~cm}$ long golden strips with a polymer cladding is used. $10 \mathrm{Gbps}$ transmission rate is reached (2.5 Gbps for each channel) at the wavelength of $1.3 \mu \mathrm{m}$. A plasmonic circuit for optical signal processing was experimentally demonstrated by [77]. The proposed structure represents an ensemble of metallic nanorods $200 \mathrm{~nm}$ in size supporting evanescently coupled LSPs. The device mixes two optical signals and provides a measure of their phase difference at the output.

\section{From Macroscopic to Microscopic Integration}

Devices and ideas discussed above are already available for implementation at the present time. Nevertheless, merging of electronics and photonics at the nanoscale is just a first step in the process which should continue in the future at a more fundamental level. The trend of miniaturization, in spite of many obstacles, unavoidably leads to molecular electronics, where single molecules are used as building blocks in devices, operated by a small amount of electrons. Integration of electronics with photonics at this level also requires single photon generation and manipulation. Description of light-matter interaction at this level leads to quantum plasmonics, among other things [78].

From a quantum mechanical point of view, volume plasmons are bosonic quasi-particles formed by collective oscillations of correlated electrons. The first quantum model is presented by Pines in 1953 [79]. The quantum model of SPPs was suggested by Elson and Ritchie in 1971 [80]. A more detailed model, which took ohmic losses into account, was introduced later by Huttner [81]. LSPs in nanoparticles were also successfully quantized [82]. Since that time many experiments were performed revealing quantum properties of plasmons including coherence, entanglement and wave-particle duality.

One of the first fundamental questions considered in quantum plasmonics is about the possibility to excite a SPP using one single photon. Experiments demonstrate that such modes exist [49]. SPPs are generated on a silver nanowire attached with nano-diamonds, where a single nitrogen-vacancy center works as a single photon emitter excited by a laser pulse. The emitted SPP then propagates along the wire and, reaching the end, is converted to a far field photon, which is subsequently detected. By accomplishing this, both wave and particle-like properties of a single SPP were discovered. In the first case, self-interference of the SPP is observed, qualitatively similar to self-interference of single photons in double slit experiments. The phase difference is provided by different distances between the emitter and one of the wire ends. Particle behavior is observed in the antibunching experiments measuring fluorescence at the wire ends. Remarkable to note is that many electrons are involved in a single SPP, but it still behaves as a single quantum particle.

SPPs reproduce many aspects of quantum behavior typical for photons. Two-photon quantum interference experiments of Hong-Ou-Mandel [83] are repeated for plasmons [84]. In the waveguided version of the experiment, a pair of photons propagates inside dielectric waveguides fabricated on a silicon chip. A directional coupler is used instead of a beam splitter to initiate the interference. To provide the conversion of photons to a SPP and back, dielectric-loaded plasmonic waveguides are integrated into ordinary dielectric waveguides. And, consequently, a plasmonic directional coupler is used instead of a dielectric one to provide the interference. Statistics at the output of two waveguides is analyzed to monitor the interference, which is clearly detected. A close correspondence between interference in dielectric and plasmonic waveguides is found. It reveals a high degree of coherence between SPP. As one of the conclusions, the authors suggest plasmonic components to be used in quantum computing.

Like photons, SPPs can be entangled (when their wave function cannot be factorized into single particle wave functions), which once again demonstrates their true quantum nature. In the experiments of [85] a pair of entangled photons (obtained using a beta barium borate nonlinear crystal, where photons are down-converted into twin photons with the doubled wavelength) passes 
through an optically thick metal film, perforated with a periodic array of subwavelength holes. Such a structure converts photons to SPP and then back to photons again at the output [86]. Coincidence counting measurements prove that the entanglement survives such a conversion. It is remarkable that no decoherence occurs between photons because of the interaction with macroscopically large amount of electrons.

Surface plasmons can exist as both macroscopic modes and purely quantum objects with just one photon involved. They can also be excited not only on macroscopic surfaces of metals, but at microscopically small objects where the features of an internal structure come into play. Quantum size effect can manifest itself as broadening, shifting and additional fine structure of the plasmon resonance [9]. Following this line of thought, it is possible to observe how at some point (some characteristic size), classical electrodynamics and the Drude model, usually used in applied plasmonics, become insufficient. This effect can be observed, for instance, in quantum materials like graphene [87]. At the typical thickness of a graphene sheet below $10 \mathrm{~nm}$, the size effect becomes dominant. Quantum effects are discovered for plasmons that are formed in subnanometre gaps between nanoparticles [88].

The described principles can be applied in the future to construct quantum plasmonic circuits [89]. The implementation of this idea meets multiple challenges. It requires small quantum emitters with efficient coupling between single photons and SPP. One elegant way using nitrogen vacancies in nano diamonds attached to silver nanowire is mentioned above [49]. Another question is how to manipulate and control SPPs at this level. This includes the development of quantum plasmonic waveguides, which should be not only small, but also homogeneous enough to guide the single SPP. Lithography has a limited precision and does not exclude impurities. One idea available in this field is the bottom-up chemical synthesis of metallic waveguides. Crystalline, smooth, defect-free structures can be obtained in this way [90]. Single plasmon detectors is another technical challenge. In [91], near field electrical interfaces are suggested to improve the detection efficiency. In their design, SPPs are converted to excitons interacting with a semiconducting nanowire. They are collected and measured in an electrical circuit as a current. The scheme is efficient enough to detect light emitted by a single quantum dot. The concept of 'dark' optoelectronic devices, where only optical near fields are used, is proposed in the paper. Obviously, to implement such ideas, one should invent a way how to transfer loss compensation and gain techniques into the quantum regime [78].

Active elements, like transistors and modulators, are expected to play a role in quantum circuits as well. Molecular transistors, operated by a small amount of electrons, are already demonstrated. Photonic transistors can be used to control the propagation of the optical signal, using another optical 'gate field' in a non-linear fashion ('gate field' may contain just a single photon). Single-photon nonlinearities are usually weak (photon interactions are rare) and such devices are hard to produce. In [92] it is proposed to use SPPs to provide strong coupling between single photons. It opens a direct way to a single-photon transistor. In the suggested theoretical model (see Figure 4) waveguided photons are converted to SPPs supported by a silver nanowire. These modes are strongly coupled to a three-level quantum gate, which can be practically realized as a quantum-dot nanocrystal or a color center in diamond. The transition from the ground state of the gate $|g\rangle$ to the excited state $|e\rangle$ is strongly coupled to the SPP. An additional metastable state $|s\rangle$ is coupled to $|e\rangle$ via an external optical control field, and decoupled from $|g\rangle$. Such a system works as an atomic memory capable of storing one photon. An incoming photon is stored if the optical control field is switched on. According to the computations, subsequent incoming signals (individual or multiple photons) can be transmitted or reflected depending on the population of the gate (similar to the photon blockade described in [93]). The described single-photon transistor can be used for both optical computations and quantum information processing. Integration of principles and devices described here with the quantum computing paradigm and distributions of functions between photons and electrons in new quantum circuits remain two important questions to be answered. 


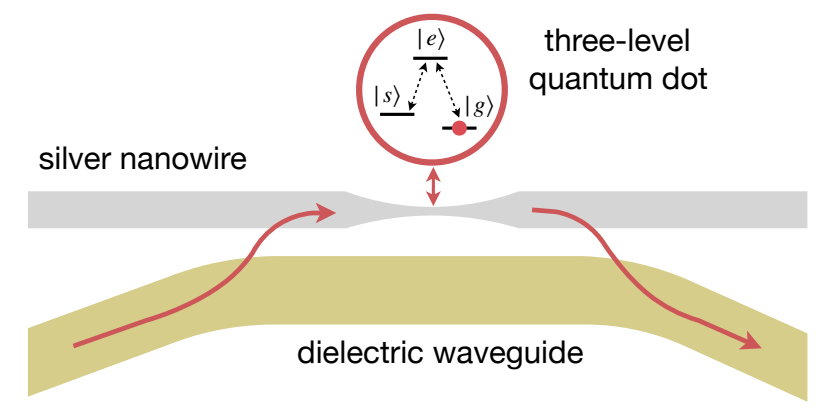

Figure 4. The design of a single-photon transistor. Reproduced from [92] with the permission. SPP modes on a silver nanowire are used to provide a strong coupling between the three-level quantum gate and waveguided photons. The gate works as a single-photon memory device controlled by the external optical field. The transparency of the transistor for the incoming signal depends on the quantum sate of the gate.

\section{More Quasiparticles}

Along with SPPs, there are other quasi-particle excitations which mix light and matter. In general, the polariton quasiparticle, introduced by Hopfield in 1958 [94], is used to describe the response of matter to light. The polarization field can be quantized and represented as an ensemble of bosons. Depending on the type of matter involved in the interaction, one may distinguish, for example, phonon-polaritons, plasmon-polaritons and exciton-polaritons. A more intense use of quasiparticles and their combinations can be fruitful approach for the deep integration between electronics and photonics.

Phonons and molecular vibrations play a significant role in many processes in nature. Interaction of electrons and photons with phonons is used a lot in modern technology. For example, the interaction of photons with molecular vibrations lay the ground for Raman scattering, which is widely used in science and industry. Interaction of electrons with molecular vibrations is an important process in biology [95-97]. It is also implemented in the resonant negative ions mass-spectrometry method to identify substances $[98,99]$. Electron-phonon interactions play a significant role in molecular electronics and is highly involved in the charge transport through molecular bridges [100,101].

Another example of useful quasiparticles with intriguing properties are exciton-polaritons, which can be formed in semiconductor microcavities. It is demonstrated that exciton-polaritons may condense at room temperatures forming a nonlinear superfluid medium [102]. Along with other types of quantum fluids, exciton-polariton condensates support many exotic excitations, including solitons and quantum vortices [103-106]. Regarding possible applications, it is already suggested theoretically and is demonstrated experimentally that nonlinear properties of exciton-polariton condensates can be implemented to create quantum simulators [107], capable of solving optimization problems with a large number of degrees of freedom.

Various quasiparticles may interact with each other forming more complex hybrid states and providing even more possibilities to use them in future electronics. Interaction of exciton-polaritons with molecular vibrations is utilized to create a prototype of a photonic transistor [108]. Another example comes from the field of plasmonic photovoltaics [109], where the solar energy of photons can be converted to electron-hole pairs in a semiconductor via the excitation of a SPP on gold nanorods. This could augment photoconversion processes and improve photon harvesting.

More experimental and theoretical works on plasmon-exciton interactions are available. In [110] coupling between excitons and SPPs is studied. The dependence of coupling on plasmonic dumping, which depends on the thickness of the plasmonic material, is demonstrated. The formation of a strongly coupled hybrid plasmon-exciton state is shown experimentally by probing the dispersion of the coupled system. The ability to tune the level of hybridization is declared to be promising for potential applications. A theoretical semi analytical model which can be used to describe coupled plasmon-exciton state is developed [111]. It describes the system where a single two-level 
quantum emitter (quantum dot or an impurity center in diamond) is coupled to an arbitrarily shaped metallic nanoparticle (silver nanorod, for instance). The model is useful for understanding and designing plasmonic-excitonic nanostructures, which potentially may find their place in novel electro-optical technologies.

\section{Conclusions}

Integration of electronics and photonics may help to overcome the existing performance-limiting factors and create the ground for the future electro-optical technologies. The deep integration assumes the usage of fundamental physical principles to provide an efficient interplay of electrons and photons. Hybrid quasiparticles mixing light and matter may help to achieve that goal. One such particle is a surface plasmon polariton. Multiple SPP-based devices, which can be used for the construction of future mixed circuits, are demonstrated theoretically and realized experimentally. One of the most important active devices, responsible for the interaction between electronic and photonic parts of a circuit, is an electro-optical modulator. SPP modes, providing subwavelength signal confinement and strong light-matter interaction, are especially useful in this class of devices. The ENZ effect in novel plasmonic materials along with the HPWG concept have proven their efficiency in various designs of modulators. The existing models technically allow the data processing frequency of the order THz. Despite the fact that most of the elements of mixed circuits are already available, the construction of large plasmonic circuits is still in its early phase. Some technical challenges, like plasmonic losses, still remain partially unsolved.

Switching from macroscopic to microscopic principles in circuit building, involving the implementation of achievements of molecular electronics and quantum plasmonics, is an upcoming task for future technologies. Manipulation of a microscopically small amount of photons and electrons to organize communication and computation requires the implementation of new paradigms and more fundamental physical principles, which may help to reconsider the existing obstacles in electronics. To organize an efficient interaction between single photons and electrons, hybrid quasiparticles may be as productive as they are in macroscopic applications. The successful implementation of plasmons and excitons hybridized with light as well as their combinations in device designing appears to be a productive approach for future technologies.

Author Contributions: The authors equally contributed to the manuscript.

Funding: This work was financially supported by the Ministry of Science and Higher Education of the Russian Federation, project No. RFMEFI58117X0026.

Conflicts of Interest: The authors declare no conflict of interest.

\section{References}

1. Thomson, D.; Zilkie, A.; Bowers, J.E.; Komljenovic, T.; Reed, G.T.; Vivien, L.; Marris-Morini, D.; Cassan, E.; Virot, L.; Fédéli, J.M.; et al. Roadmap on silicon photonics. J. Opt. 2016, 18, 073003. [CrossRef]

2. Su, T.A.; Neupane, M.; Steigerwald, M.L.; Venkataraman, L.; Nuckolls, C. Chemical principles of single-molecule electronics. Nat. Rev. Mater. 2016, 1, 16002. [CrossRef]

3. Stockman, M.I.; Kneipp, K.; Bozhevolny, S.I.; Saha, S.; Dutta, A.; Ndukaife, J.; Kinsey, N.; Reddy, H.; Guler, U.; Shalaev, V.M.; et al. Roadmap on plasmonics. J. Opt. 2018, 20, 043001. [CrossRef]

4. Novotny, L.; Hecht, B. Principles of Nano-Optics; Cambridge University Press: Cambridge, UK, 2012.

5. Maier, S.A. Plasmonics: Fundamentals and Applications; Springer Science \& Business Media: New York, NY, USA, 2007.

6. Agranovich, V.; Mills, D. Surface Polaritons; North-Holland Publishing Company: New York, NY, USA, 1982.

7. Ritchie, R.H. Plasma losses by fast electrons in thin films. Phys. Rev. 1957, 106, 874. [CrossRef]

8. Powell, C.; Swan, J. Effect of oxidation on the characteristic loss spectra of aluminum and magnesium. Phys. Rev. 1960, 118, 640. [CrossRef]

9. Scholl, J.A.; Koh, A.L.; Dionne, J.A. Quantum plasmon resonances of individual metallic nanoparticles. Nature 2012, 483, 421. [CrossRef] [PubMed] 
10. Pitarke, J.; Silkin, V.; Chulkov, E.; Echenique, P. Theory of surface plasmons and surface-plasmon polaritons. Rep. Prog. Phys. 2006, 70, 1. [CrossRef]

11. Nie, S.; Emory, S.R. Probing single molecules and single nanoparticles by surface-enhanced Raman scattering. Science 1997, 275, 1102-1106. [CrossRef] [PubMed]

12. Kneipp, K.; Wang, Y.; Kneipp, H.; Perelman, L.T.; Itzkan, I.; Dasari, R.R.; Feld, M.S. Single molecule detection using surface-enhanced Raman scattering (SERS). Phys. Rev. Lett. 1997, 78, 1667. [CrossRef]

13. Liu, N.; Tang, M.L.; Hentschel, M.; Giessen, H.; Alivisatos, A.P. Nanoantenna-enhanced gas sensing in a single tailored nanofocus. Nat. Mater. 2011, 10, 631. [CrossRef] [PubMed]

14. Stockman, M.I. Nanoplasmonic sensing and detection. Science 2015, 348, 287-288. [CrossRef] [PubMed]

15. Barnes, W.L.; Dereux, A.; Ebbesen, T.W. Surface plasmon subwavelength optics. Nature 2003, 424, 824. [CrossRef] [PubMed]

16. Ozbay, E. Plasmonics: Merging photonics and electronics at nanoscale dimensions. Science 2006, 311, $189-193$. [CrossRef] [PubMed]

17. Gramotnev, D.K.; Bozhevolnyi, S.I. Plasmonics beyond the diffraction limit. Nat. Photonics 2010, 4, 83. [CrossRef]

18. Stockman, M.I. Nanoplasmonics: Past, present, and glimpse into future. Opt. Express 2011, 19, $22029-22106$. [CrossRef] [PubMed]

19. Davis, T.J.; Gómez, D.E.; Roberts, A. Plasmonic circuits for manipulating optical information. Nanophotonics 2017, 6, 543-559. [CrossRef]

20. Liu, K.; Ye, C.R.; Khan, S.; Sorger, V.J. Review and perspective on ultrafast wavelength-size electro-optic modulators. Laser Photonics Rev. 2015, 9, 172-194. [CrossRef]

21. Johnson, P.B.; Christy, R.W. Optical constants of the noble metals. Phys. Rev. B 1972, 6, 4370. [CrossRef]

22. West, P.R.; Ishii, S.; Naik, G.V.; Emani, N.K.; Shalaev, V.M.; Boltasseva, A. Searching for better plasmonic materials. Laser Photonics Rev. 2010, 4, 795-808. [CrossRef]

23. Naik, G.V.; Shalaev, V.M.; Boltasseva, A. Alternative Plasmonic Materials: Beyond Gold and Silver. Adv. Mater. 2013, 25, 3264-3294. [CrossRef] [PubMed]

24. Bhatta, H.L.; Aliev, A.E.; Drachev, V.P. New mechanism of plasmons specific for spin-polarized nanoparticles. Sci. Rep. 2019, 9. [CrossRef] [PubMed]

25. Soref, R.; Peale, R.E.; Buchwald, W. Longwave plasmonics on doped silicon and silicides. Opt. Express 2008, 16, 6507-6514. [CrossRef] [PubMed]

26. Dionne, J.A.; Sweatlock, L.A.; Sheldon, M.T.; Alivisatos, A.P.; Atwater, H.A. Silicon-based plasmonics for on-chip photonics. IEEE J. Sel. Top. Quantum Electron. 2010, 16, 295-306. [CrossRef]

27. Noginov, M.; Gu, L.; Livenere, J.; Zhu, G.; Pradhan, A.; Mundle, R.; Bahoura, M.; Barnakov, Y.A.; Podolskiy, V. Transparent conductive oxides: Plasmonic materials for telecom wavelengths. Appl. Phys. Lett. 2011, 99, 021101. [CrossRef]

28. Exarhos, G.J.; Zhou, X.D. Discovery-based design of transparent conducting oxide films. Thin Solid Films 2007, 515, 7025-7052. [CrossRef]

29. Kulkarni, A.; Knickerbocker, S. Estimation and verification of the electrical properties of indium tin oxide based on the energy band diagram. J. Vac. Sci. Technol. A Vac. Surf. Films 1996, 14, 1709-1713. [CrossRef]

30. Michelotti, F.; Dominici, L.; Descrovi, E.; Danz, N.; Menchini, F. Thickness dependence of surface plasmon polariton dispersion in transparent conducting oxide films at $1.55 \mu \mathrm{m}$. Opt. Lett. 2009, 34, 839-841. [CrossRef] [PubMed]

31. Franzen, S.; Rhodes, C.; Cerruti, M.; Gerber, R.W.; Losego, M.; Maria, J.P.; Aspnes, D. Plasmonic phenomena in indium tin oxide and ITO-Au hybrid films. Opt. Lett. 2009, 34, 2867-2869. [CrossRef] [PubMed]

32. Niu, X.; Hu, X.; Chu, S.; Gong, Q. Epsilon-Near-Zero Photonics: A New Platform for Integrated Devices. Adv. Opt. Mater. 2018, 6, 1701292. [CrossRef]

33. Caspani, L.; Kaipurath, R.; Clerici, M.; Ferrera, M.; Roger, T.; Kim, J.; Kinsey, N.; Pietrzyk, M.; Di Falco, A.; Shalaev, V.; et al. Enhanced Nonlinear Refractive Index in $\epsilon$-Near-Zero Materials. Phys. Rev. Lett. 2016, 116, 233901. [CrossRef] [PubMed]

34. Alam, M.; De Leon, I.; Boyd, R. Large optical nonlinearity of indium tin oxide in its epsilon-near-zero region. Science 2016, 352, 795-797. [CrossRef] [PubMed]

35. Chen, C.; Wang, Z.; Wu, K.; Ye, H. Tunable near-infrared epsilon-near-zero and plasmonic properties of Ag-ITO co-sputtered composite films. Sci. Technol. Adv. Mater. 2018, 19, 174-184. [CrossRef] [PubMed] 
36. Fang, X.; Mak, C.L.; Dai, J.; Li, K.; Ye, H.; Leung, C.W. ITO/Au/ITO sandwich structure for near-infrared plasmonics. ACS Appl. Mater. Interfaces 2014, 6, 15743-15752. [CrossRef] [PubMed]

37. Pollard, R.; Murphy, A.; Hendren, W.; Evans, P.; Atkinson, R.; Wurtz, G.; Zayats, A.; Podolskiy, V.A. Optical nonlocalities and additional waves in epsilon-near-zero metamaterials. Phys. Rev. Lett. 2009, 102, 127405. [CrossRef] [PubMed]

38. Ju, L.; Geng, B.; Horng, J.; Girit, C.; Martin, M.; Hao, Z.; Bechtel, H.A.; Liang, X.; Zettl, A.; Shen, Y.R.; et al. Graphene plasmonics for tunable terahertz metamaterials. Nat. Nanotechnol. 2011, 6, 630-634. [CrossRef] [PubMed]

39. Yan, H.; Li, X.; Chandra, B.; Tulevski, G.; Wu, Y.; Freitag, M.; Zhu, W.; Avouris, P.; Xia, F. Tunable infrared plasmonic devices using graphene/insulator stacks. Nat. Nanotechnol. 2012, 7, 330-334. [CrossRef] [PubMed]

40. Chen, J.; Badioli, M.; Alonso-González, P.; Thongrattanasiri, S.; Huth, F.; Osmond, J.; Spasenović, M.; Centeno, A.; Pesquera, A.; Godignon, P.; et al. Optical nano-imaging of gate-tunable graphene plasmons. Nature 2012, 487, 77. [CrossRef] [PubMed]

41. Fei, Z.; Rodin, A.; Andreev, G.; Bao, W.; McLeod, A.; Wagner, M.; Zhang, L.; Zhao, Z.; Thiemens, M.; Dominguez, G.; et al. Gate-tuning of graphene plasmons revealed by infrared nano-imaging. Nature 2012, 487, 82. [CrossRef] [PubMed]

42. Grigorenko, A.; Polini, M.; Novoselov, K. Graphene plasmonics. Nat. Photonics 2012, 6, 749. [CrossRef]

43. Ni, G.; McLeod, A.; Sun, Z.; Wang, L.; Xiong, L.; Post, K.; Sunku, S.; Jiang, B.Y.; Hone, J.; Dean, C.R.; et al. Fundamental limits to graphene plasmonics. Nature 2018, 557, 530. [CrossRef] [PubMed]

44. Wilk, G.D.; Wallace, R.M.; Anthony, J. High- $\kappa$ gate dielectrics: Current status and materials properties considerations. J. Appl. Phys. 2001, 89, 5243-5275. [CrossRef]

45. Robertson, J. High dielectric constant oxides. Eur. Phys. J. Appl. Phys. 2004, 28, 265-291. [CrossRef]

46. Bohr, M.T.; Chau, R.S.; Ghani, T.; Mistry, K. The high-k solution. IEEE Spectr. 2007, 44, 29-35. [CrossRef]

47. Okamoto, K.; Niki, I.; Shvartser, A.; Narukawa, Y.; Mukai, T.; Scherer, A. Surface-plasmon-enhanced light emitters based on InGaN quantum wells. Nat. Mater. 2004, 3, 601. [CrossRef] [PubMed]

48. Han, Z.; Bozhevolnyi, S.I. Radiation guiding with surface plasmon polaritons. Rep. Prog. Phys. 2012, 76, 016402. [CrossRef] [PubMed]

49. Kolesov, R.; Grotz, B.; Balasubramanian, G.; Stöhr, R.J.; Nicolet, A.A.; Hemmer, P.R.; Jelezko, F.; Wrachtrup, J. Wave-particle duality of single surface plasmon polaritons. Nat. Phys. 2009, 5, 470. [CrossRef]

50. Alam, M.Z.; Aitchison, J.S.; Mojahedi, M. A marriage of convenience: Hybridization of surface plasmon and dielectric waveguide modes. Laser Photonics Rev. 2014, 8, 394-408. [CrossRef]

51. Nezhad, M.P.; Tetz, K.; Fainman, Y. Gain assisted propagation of surface plasmon polaritons on planar metallic waveguides. Opt. Express 2004, 12, 4072-4079. [CrossRef] [PubMed]

52. Noginov, M.; Podolskiy, V.A.; Zhu, G.; Mayy, M.; Bahoura, M.; Adegoke, J.; Ritzo, B.; Reynolds, K. Compensation of loss in propagating surface plasmon polariton by gain in adjacent dielectric medium. Opt. Express 2008, 16, 1385-1392. [CrossRef] [PubMed]

53. Grandidier, J.; Des Francs, G.C.; Massenot, S.; Bouhelier, A.; Markey, L.; Weeber, J.C.; Finot, C.; Dereux, A. Gain-assisted propagation in a plasmonic waveguide at telecom wavelength. Nano Lett. 2009, 9, 2935-2939. [CrossRef] [PubMed]

54. De Leon, I.; Berini, P. Amplification of long-range surface plasmons by a dipolar gain medium. Nat. Photonics 2010, 4, 382. [CrossRef]

55. Bergman, D.J.; Stockman, M.I. Surface plasmon amplification by stimulated emission of radiation: Quantum generation of coherent surface plasmons in nanosystems. Phys. Rev. Lett. 2003, 90, 027402. [CrossRef] [PubMed]

56. Galanzha, E.I.; Weingold, R.; Nedosekin, D.A.; Sarimollaoglu, M.; Nolan, J.; Harrington, W.; Kuchyanov, A.S.; Parkhomenko, R.G.; Watanabe, F.; Nima, Z.; et al. Spaser as a biological probe. Nat. Commun. 2017, 8, 15528. [CrossRef] [PubMed]

57. Melikyan, A.; Lindenmann, N.; Walheim, S.; Leufke, P.; Ulrich, S.; Ye, J.; Vincze, P.; Hahn, H.; Schimmel, T.; Koos, C.; et al. Surface plasmon polariton absorption modulator. Opt. Express 2011, 19, 8855-8869. [CrossRef] [PubMed]

58. Neira, A.D.; Wurtz, G.A.; Zayats, A.V. All-optical switching in silicon photonic waveguides with an epsilon-near-zero resonant cavity. Photonics Res. 2018, 6, B1-B5. [CrossRef]

59. Qiu, X.; Ruan, X.; Li, Y.; Zhang, F. Indium Tin Oxide Based Dual-Polarization Electro-Optic Intensity Modulator on a Single Silicon Waveguide. J. Lightw. Technol. 2018, 36, 2563-2571. [CrossRef] 
60. Sinatkas, G.; Pitilakis, A.; Zografopoulos, D.C.; Beccherelli, R.; Kriezis, E.E. Transparent conducting oxide electro-optic modulators on silicon platforms: A comprehensive study based on the drift-diffusion semiconductor model. J. Appl. Phys. 2017, 121, 023109. [CrossRef]

61. Vasudev, A.P.; Kang, J.H.; Park, J.; Liu, X.; Brongersma, M.L. Electro-optical modulation of a silicon waveguide with an "epsilon-near-zero" material. Opt. Express 2013, 21, 26387-26397. [CrossRef] [PubMed]

62. Zografopoulos, D.; Sinatkas, G.; Lotfi, E.; Shahada, L.; Swillam, M.; Kriezis, E.; Beccherelli, R. Amplitude modulation in infrared metamaterial absorbers based on electro-optically tunable conducting oxides. Appl. Phys. A 2018, 124, 105. [CrossRef]

63. Ayata, M.; Fedoryshyn, Y.; Heni, W.; Baeuerle, B.; Josten, A.; Zahner, M.; Koch, U.; Salamin, Y.; Hoessbacher, C.; Haffner, C.; et al. High-speed plasmonic modulator in a single metal layer. Science 2017, 358, 630-632. [CrossRef] [PubMed]

64. Dionne, J.A.; Diest, K.; Sweatlock, L.A.; Atwater, H.A. PlasMOStor: A metal- oxide- Si field effect plasmonic modulator. Nano Lett. 2009, 9, 897-902. [CrossRef] [PubMed]

65. Haffner, C.; Chelladurai, D.; Fedoryshyn, Y.; Josten, A.; Baeuerle, B.; Heni, W.; Watanabe, T.; Cui, T.; Cheng, B.; Saha, S.; et al. Low-loss plasmon-assisted electro-optic modulator. Nature 2018, 556, 483. [CrossRef] [PubMed]

66. Lee, H.W.; Papadakis, G.; Burgos, S.P.; Chander, K.; Kriesch, A.; Pala, R.; Peschel, U.; Atwater, H.A. Nanoscale conducting oxide PlasMOStor. Nano Lett. 2014, 14, 6463-6468. [CrossRef] [PubMed]

67. Nikolajsen, T.; Leosson, K.; Bozhevolnyi, S.I. Surface plasmon polariton based modulators and switches operating at telecom wavelengths. Appl. Phys. Lett. 2004, 85, 5833-5835. [CrossRef]

68. Sorger, V.J.; Lanzillotti-Kimura, N.D.; Ma, R.M.; Zhang, X. Ultra-compact silicon nanophotonic modulator with broadband response. Nanophotonics 2012, 1, 17-22. [CrossRef]

69. Lu, Z.; Zhao, W.; Shi, K. Ultracompact electroabsorption modulators based on tunable epsilon-near-zero-slot waveguides. IEEE Photonics J. 2012, 4, 735-740.

70. Amin, R.; Khurgin, J.B.; Sorger, V.J. Waveguide-based electro-absorption modulator performance: Comparative analysis. Opt. Express 2018, 26, 15446. [CrossRef] [PubMed]

71. Gao, L.; Huo, Y.; Harris, J.S.; Zhou, Z. Ultra-compact and low-loss polarization rotator based on asymmetric hybrid plasmonic waveguide. IEEE Photonics Technol. Lett. 2013, 25, 2081-2084. [CrossRef]

72. Kim, S.; Qi, M. Polarization rotation and coupling between silicon waveguide and hybrid plasmonic waveguide. Opt. Express 2015, 23, 9968-9978. [CrossRef] [PubMed]

73. Majumder, A.; Shen, B.; Polson, R.; Menon, R. Ultra-compact polarization rotation in integrated silicon photonics using digital metamaterials. Opt. Express 2017, 25, 19721-19731. [CrossRef] [PubMed]

74. Pshenichnyuk, I.A.; Kosolobov, S.S.; Maimistov, A.I.; Drachev, V.P. Conversion of light polarisation in asymmetric plasmonic waveguides. Quantum Electron. 2018, 48, 1153. [CrossRef]

75. An, S.; Kwon, O.K. Integrated InP polarization rotator using the plasmonic effect. Opt. Express 2018, 26, 1305-1314. [CrossRef] [PubMed]

76. Kim, J.T.; Ju, J.J.; Park, S.; Kim, M.S.; Park, S.K.; Lee, M.H. Chip-to-chip optical interconnect using gold long-range surface plasmon polariton waveguides. Opt. Express 2008, 16, 13133-13138. [CrossRef] [PubMed]

77. Eftekhari, F.; Gómez, D.E.; Davis, T.J. Measuring subwavelength phase differences with a plasmonic circuit-An example of nanoscale optical signal processing. Opt. Lett. 2014, 39, 2994-2997. [CrossRef] [PubMed]

78. Tame, M.S.; McEnery, K.; Özdemir, Ş.; Lee, J.; Maier, S.; Kim, M. Quantum plasmonics. Nat. Phys. 2013, 9, 329. [CrossRef]

79. Pines, D. A collective description of electron interactions: IV. Electron interaction in metals. Phys. Rev. 1953, 92, 626. [CrossRef]

80. Elson, J.; Ritchie, R. Photon interactions at a rough metal surface. Phys. Rev. B 1971, 4, 4129. [CrossRef]

81. Huttner, B.; Barnett, S.M. Quantization of the electromagnetic field in dielectrics. Phys. Rev. A 1992, 46, 4306. [CrossRef] [PubMed]

82. Crowell, J.; Ritchie, R. Radiative decay of Coulomb-stimulated plasmons in spheres. Phys. Rev. 1968, 172, 436. [CrossRef]

83. Hong, C.K.; Ou, Z.Y.; Mandel, L. Measurement of subpicosecond time intervals between two photons by interference. Phys. Rev. Lett. 1987, 59, 2044. [CrossRef] [PubMed]

84. Fakonas, J.S.; Lee, H.; Kelaita, Y.A.; Atwater, H.A. Two-plasmon quantum interference. Nat. Photonics 2014, 8, 317. [CrossRef] 
85. Altewischer, E.; Van Exter, M.; Woerdman, J. Plasmon-assisted transmission of entangled photons. Nature 2002, 418, 304. [CrossRef] [PubMed]

86. Ebbesen, T.W.; Lezec, H.J.; Ghaemi, H.; Thio, T.; Wolff, P.A. Extraordinary optical transmission through sub-wavelength hole arrays. Nature 1998, 391, 667. [CrossRef]

87. Thongrattanasiri, S.; Manjavacas, A.; García de Abajo, F.J. Quantum finite-size effects in graphene plasmons. Acs Nano 2012, 6, 1766-1775. [CrossRef] [PubMed]

88. Zhu, W.; Esteban, R.; Borisov, A.G.; Baumberg, J.J.; Nordlander, P.; Lezec, H.J.; Aizpurua, J.; Crozier, K.B. Quantum mechanical effects in plasmonic structures with subnanometre gaps. Nat. Commun. 2016, 7, 11495. [CrossRef] [PubMed]

89. De Leon, N.P.; Lukin, M.D.; Park, H. Quantum plasmonic circuits. IEEE J. Sel. Top. Quantum Electron. 2012, 18, 1781-1791. [CrossRef]

90. Wiley, B.; Sun, Y.; Xia, Y. Polyol synthesis of silver nanostructures: control of product morphology with Fe (II) or Fe (III) species. Langmuir 2005, 21, 8077-8080. [CrossRef] [PubMed]

91. Falk, A.L.; Koppens, F.H.; Chun, L.Y.; Kang, K.; de Leon Snapp, N.; Akimov, A.V.; Jo, M.H.; Lukin, M.D.; Park, H. Near-field electrical detection of optical plasmons and single-plasmon sources. Nat. Phys. 2009, 5, 475. [CrossRef]

92. Chang, D.E.; Sørensen, A.S.; Demler, E.A.; Lukin, M.D. A single-photon transistor using nanoscale surface plasmons. Nat. Phys. 2007, 3, 807. [CrossRef]

93. Birnbaum, K.M.; Boca, A.; Miller, R.; Boozer, A.D.; Northup, T.E.; Kimble, H.J. Photon blockade in an optical cavity with one trapped atom. Nature 2005, 436, 87. [CrossRef] [PubMed]

94. Hopfield, J. Theory of the contribution of excitons to the complex dielectric constant of crystals. Phys. Rev. 1958, 112, 1555. [CrossRef]

95. Pshenichnyuk, S.A.; Rakhmeyev, R.G.; Asfandiarov, N.L.; Komolov, A.S.; Modelli, A.; Jones, D. Can the Electron-Accepting Properties of Odorants Be Involved in Their Recognition by the Olfactory System? J. Phys. Chem. Lett. 2018, 9, 2320-2325. [CrossRef] [PubMed]

96. Pshenichnyuk, S.A.; Modelli, A.; Komolov, A.S. Interconnections between dissociative electron attachment and electron-driven biological processes. Int. Rev. Phys. Chem. 2018, 37, 125-170. [CrossRef]

97. Pshenichnyuk, S.A.; Modelli, A.; Vorob'ev, A.S.; Asfandiarov, N.L.; Nafikova, E.P.; Rakhmeyev, R.G.; Galeev, R.V.; Komolov, A.S. Fragmentation of chlorpyrifos by thermal electron attachment: A likely relation to its metabolism and toxicity. Phys. Chem. Chem. Phys. 2018, 20, 22272-22283. [CrossRef] [PubMed]

98. Pshenichnyuk, S.; Pshenichnyuk, I.; Nafikova, E.; Asfandiarov, N. Dissociative electron attachment in selected haloalkanes. Rapid Commun. Mass Spectrom. 2006, 20, 1097-1103. [CrossRef] [PubMed]

99. Pshenichnyuk, S.; Lomakin, G.; Fokin, A.; Pshenichnyuk, I.; Asfandiarov, N. Temperature dependence of the mean autodetachment lifetime of the p-benzoquinone molecular radical anion. Rapid Commun. Mass Spectrom. 2006, 20, 383-386. [CrossRef] [PubMed]

100. Pshenichnyuk, I. Interaction of Electrons with Vibrating Molecules: Molecular Electronic Applications; LAP LAMBERT Academic Publishing: Riga, Latvia, 2012.

101. Pshenichnyuk, I.A.; Čížek, M. Motor effect in electron transport through a molecular junction with torsional vibrations. Phys. Rev. B 2011, 83, 165446. [CrossRef]

102. Lagoudakis, K. The Physics of Exciton-Polariton Condensates; EPFL Press: Lausanne, Switzerland, 2013.

103. Pshenichnyuk, I.A. Pressure-induced vortex rings multiplication as a source of vorticity in superfluids. Lett. Mater. 2015, 5, 385-388. [CrossRef]

104. Pshenichnyuk, I.A.; Berloff, N.G. Inelastic scattering of xenon atoms by quantized vortices in superfluids. Phys. Rev. B 2016, 94, 184505. [CrossRef]

105. Pshenichnyuk, I. Static and dynamic properties of heavily doped quantum vortices. New J. Phys. 2017, 19, 105007. [CrossRef]

106. Pshenichnyuk, I.A. Pair interactions of heavy vortices in quantum fluids. Phys. Lett. A 2018, 382, $523-527$. [CrossRef]

107. Berloff, N.G.; Silva, M.; Kalinin, K.; Askitopoulos, A.; Töpfer, J.D.; Cilibrizzi, P.; Langbein, W.; Lagoudakis, P.G. Realizing the classical XY Hamiltonian in polariton simulators. Nat. Mater. 2017, 16, 1120. [CrossRef] [PubMed]

108. Zasedatelev, A.V.; Baranikov, A.V.; Urbonas, D.; Scafirimuto, F.; Scherf, U.; Stöferle, T.; Mahrt, R.F.; Lagoudakis, P.G. A room-temperature organic polariton transistor. Nat. Photonics 2019, 13, 378. [CrossRef] 
109. Mubeen, S.; Lee, J.; Singh, N.; Krämer, S.; Stucky, G.D.; Moskovits, M. An autonomous photosynthetic device in which all charge carriers derive from surface plasmons. Nat. Nanotechnol. 2013, 8, 247. [CrossRef] [PubMed]

110. Balci, S.; Kocabas, C.; Ates, S.; Karademir, E.; Salihoglu, O.; Aydinli, A. Tuning surface plasmon-exciton coupling via thickness dependent plasmon damping. Phys. Rev. B 2012, 86, 235402. [CrossRef]

111. Gómez, D.E.; Roberts, A.; Davis, T.J.; Vernon, K.C. Surface plasmon hybridization and exciton coupling. Phys. Rev. B 2012, 86, 035411. [CrossRef]

(C) 2019 by the authors. Licensee MDPI, Basel, Switzerland. This article is an open access article distributed under the terms and conditions of the Creative Commons Attribution (CC BY) license (http://creativecommons.org/licenses/by/4.0/). 